\title{
Listados de Posters
}

1989

Titulo: IMPORTANCIA DE LA ORGANIZACIÓN DE ATENEOS DE SALUD PÚBLICA MINISTERIO DE SALUD AÑO 2016

Autores: AREDES R., MUGAS ORTIZ A., AREDES S. MINISTERIO DE SALUD DE CATAMARCA

1990

Titulo: ESTUDIO DE LA PREVALENCIA DE CARIES DENTAL Y SU RELACIÓN ENTRE EL CONSUMO DE PRODUCTOS AZUCARADOS Y LAS PRÁCTICAS DE AUTO-CUIDADO EN SALUD BUCAL, EN ESTUDIANTES DE LA UNIVERSIDAD NACIONAL DE CÓRDOBA

Autores: SANZ A., FLORES V., SICA SANCHEZ N.

1991

Titulo: FOMENTAR EL PROCESO SALUD-ENFERMEDAD-ATENCIÓN BUCAL EN ESTUDIANTES SECUNDARIOS DE CÓRDOBA

Autores: FLORES V., SANZ A., SICA SANCHEZ N.

1992

Titulo: ACTIVIDAD EXTENSIONISTA INTER-FACULTADES: "QUE BICHO ES EL ALACRÁN"

Autores: PONS A., MIRANDA A., MENARDI F., URIBARREN C., PONCE J., DAGUERO M. 
Titulo: ESTRATEGIAS DE SALUD PARA HACER FRENTE A UNA SOCIEDAD QUE ENVEJECE EN LA CIUDAD DE CATAMARCA AÑO 2016-2018

Autores: AREDES R., BARRIONUEVO A., CARRIZO L., CORDOBA J., ZEBALLOS J., SOSA R., REARTES C.

2004

Titulo: FASCIOLOSIS ENDÉMICA EN CATAMARCA: CARACTERIZACIÓN EN HUMANOS, ANIMALES Y LIMNEIDOS. SUPERACIÓN DE PROBLEMAS DE DIAGNÓSTICO

Autores: MALANDRINI J., CARNEVALE S., VELAZQUEZ J., SALAS L., CORDOBA P., KAMENETZKY L., MARTINEZ M., PANTANO M., SORIA C. FACULTAD DE CIENCIAS DE LASALUD. UNIVERSIDAD NACIONAL DE CATAMARCA. CATAMARCA, ARGENTINA.

2006

Titulo: DIFUSIÓN DE LA IMPORTANCIA SANITARIA DE LOS ARTRÓPODOS

Autores: FERRERO M., SÁNCHEZ R., FERNÁNDEZ G., ASÍS E., PIZZI R., PIZZI $\mathrm{H}$.

2009

Titulo: CAMPAÑA DE PREVENCIÓN DEL CÁNCER BUCAL "SACALE LA LENGUA AL CÁNCER". EXPERIENCIA 2016

Autores: BACHMEIER E., ALLENDE A., BELARDINELLI P., BOLESINA N., BRUSA M., CACIVA R., CARRICA A., CRISCUOLO I., GALÍNDEZ F., GILLIGAN G., LAZOS J., LIANDRO F., MOINE L., MORELATTO R., RÍOS F., ROBLEDO G., RODRIGUEZ MARCO E., SECCHI D., TALAVERA A., VARGAS J., ZAPATA M., PANICO R., PIEMONTE E., LÓPEZ DE BLANC S.

2010

Titulo: VALORACIONES SOBRE LA INSERCIÓN EN EL SISTEMA DE SALUD DE LOS PROFESIONALES ODONTÓLOGOS

Autores: OSILIO M., ZAMAR S.

2011

Titulo: MEDIDAS DE BIOSEGURIDAD EN LA PRÁCTICA ODONTOLÓGICA EN EL AÑO 2017

Autores: MENDOZA F., STROPA G., ZAMAR A.

2013

Titulo: ADMINISTRACIÓN ESTATAL DE LA VIDA Y DISTRIBUCIÓN DIFERENCIAL DEL ACCESO A LA SALUD

Autores: HERRANZ M. 
2017

Titulo: ALIMENTOS FUNCIONALES: PERCEPCION DEL CONSUMIDOR Y MENSAJES PUBLICITARIOS VS. EVIDENCIA CIENTIFICA

Autores: BASSANI A., FORSYTH M., APARICIO S., SANCHEZ S., PERALTA M., NAVARRO A., COSTAS CZARNECKI P.

2018

Titulo: EFECTO DE LA CONTAMINACIÓN ATMOSFÉRICA SOBRE LA SALUD INFANTIL

Autores: ORCELLET E., NOIR J., SAVOY F., VILLANOVA M., DE GRACIAL.

2019

Titulo: VIGILANCIA ENTOMOLÓGICA DE AEDES AEGYPTI: UNA ESTRATEGIA DE ENSEÑANZA-APRENDIZAJE

Autores: CASTRO M., CARRANZA M., OBERTO S., RIZOS P., UGNIA L., RICHARDET M., GOMEZ C.

\begin{abstract}
2021
Titulo: LIBRO INFANTIL: MANOS LIMPIAS. NIÑOS MÁS SANOS

Autores: OBERTO M., FERRERO M., MAMONDI V., VACA NARVAJA S., SÁNCHEZ R.
\end{abstract}

2022

Titulo: EXPERIENCIAS SIGNIFICATIVAS DE LOS INTEGRANTES DE LA ESCUELA DE ALIMENTOS Y DESARROLLO HUMANO DE LA UNIVERSIDAD DE ANTIOQUIA: UN APORTE A LA SEGURIDAD ALIMENTARIA EN COMUNIDADES VULNERABLES DE LA CIUDAD DE MEDELLÍN

Autores: RODRIGUEZ ECHAVARRIA D., CATAÑO MONTOYA Y., JARAMILLO ROJAS L.

\title{
2023
}

Titulo: REDUCIENDO LOS RIESGOS PARA LA SALUD EN LA PRODUCCIÓN DE GRABADOS EN ARTES VISUALES

Autores: VILLAGRAN C., MIRANDA A., PEDREROL L., PONS A., MARGARA M., MADELON N., SÁNCHEZ S., FERNANDEZ L., BONGIOVANI A., DAGHERO A., CANESINI B., LIZARRAGA C.

2024

Titulo: PROYECTO RECOBA VILLAGUAY

Autores: BELMONTE V., GERVASONI A., ETCHEVERRY G., HORISBERGER G., RODRIGUEZ E., CANOVAS J., MICHEL J., CABANNA G., PIACENZA G., GILBERTO J., WENDLER S., TORREZ M., AZARIO R. 
2026

Titulo: CENTRO DE PRÁCTICAS EN SALUD PÚBLICA DE LA ESCUELA DE NUTRICIÓN EN EL ÁMBITO UNIVERSITARIO

Autores: BARBERO L., SCRUZZI G., EPPENS M., MARTINA D., BOCCO R.

2030

Titulo: EVALUACION DEL ESTADO NUTRICIONAL Y PERFIL ALIMENTARIO DE PERSONAS CON DIETAS VEGETARIANAS

Autores: CASERMEIRO A., GASPARINI S., GILOBERT M.

FACULTAD DE CIENCIAS DE LA SALUD. UNIVERSIDAD NACIONAL

DE SALTA. SALTA. ARGENTINA

2040

Titulo: EXPERIENCIA DEL EQUIPO INTERDISCIPLINARIO DE SALUD EN USO DE TEST RAPIDOS DE VIH

Autores: OEXLER I., RODRIGUEZ S., HERRERA C., BOUCHET D., BARBAS G., BALANGERO $M$.

2044

Titulo: HOSPITALES SOSTENIBLES: LINEA DE BASE DE INDICADORES DE SOSTENIBILIDAD AMBIENTAL

Autores: SMITH M., CORREA G.

2046

Titulo: PROGRAMA CUS-SUMAR, UNA EXPERIENCIA DE GESTIÓN EN SALUD PÚBLICA EN LA PROVINCIA DE CÓRDOBA

Autores: MOSCATELLO E., FONCEA L., OLMOS M., PORUCINI M., CARBÓ C., CALVO D., TORRES M.

COBERTURA UNIVERSAL DE SALUD - SUMAR, CÓRDOBA, ARGENTINA

2051

Titulo: IMPLEMENTACIÓN DE DIAGNÓSTICO MOLECULAR EN LABORATORIO DE SALUD PÚBLICA: OBSTÁCULOS Y BENEFICIOS Autores: ESTEVES B., VERDUGO R., TALIA J.

2052

Titulo: PLAN DE GESTIÓN DE RIESGOS DEL LABORATORIO DE HEMODERIVADOS UNC: IMPACTO EN SALUD PÚBLICA

Autores: BARROS C., RIVERO R., MASSA C., FONTANA D. 
2056

Titulo: ARTICULACIÓN INTERINSTITUCIONAL PARA UNA INTERVENCIÓN COMUNITARIA: "MOSQUITO TE VAMO'A KUSHPILLAR"

Autores: DOMÍNGUEZ A., PAZ M., VALDEZ M., DOMINGUEZ L.

2059

Titulo: PROMOCIÓN DE LA SALUD INTEGRAL. PREVENCIÓN DEL DENGUE, ZIKA Y CHIKUNGUNYA

Autores: GILARDI A., SALVATIERRA E., SUBELZA R., GROH M.

2060

Titulo: INVESTIGACIÓN ACCIÓN CONSTRUYENDO ÁMBITOS Y PRÁCTICAS SALUDABLES

Autores: SALVATIERRA E., GILARDI A., SUBELZA R., GROH M.

2069

Titulo: NIVEL DE ACTIVIDAD FÍSICA SISTEMÁTICA DE UN GRUPO DE ALUMNOS DE LA ASIGNATURA FISIOLOGÍA HUMANA

Autores: LLERMANOS G.

2070

Titulo: ESTADO NUTRICIONAL DE NIÑOS Y SU RELACIÓN CON ANTECEDENTES PERINATALES Y NIVEL SOCIOECONÓMICO FAMILIAR. CÓRDOBA

Autores: BISCO M., RAMÍREZ R., BORGONOVO M., BERRA S., REARTES G.

2073

Titulo: DESIGUALDADES SOCIALES Y SALUD: HACIALACONFORMACIÓN DEL OBSERVATORIO SOCIO-SANITARIO PARA CONCEPCIÓN DEL URUGUAY

Autores: OLIVAM., DE GRACIAL., GARCIAA., SAVOY F., PUSCHP., RETAMARS.

\section{7}

Titulo: EXPLORACIÓN DE CONDICIONES Y REPRESENTACIONES DE USUARIOS DE CAPS. UN ESTUDIO DE ACCESIBILIDAD

Autores: DE GRACIA L., SAVOY F., GARCÍA A., RETAMAR S., MULA D., GERVASONI A., DE BATTISTAA.

2080

Titulo: EVALUACIÓN Y PREVENCIÓN DEL DETERIORO COGNITIVO EXPERIENCIA DEL SERVICIO DE NEUROPSICOLOGÍA (FACULTAD DE PSICOLOGÍA)

Autores: BASTIDA M., MÍAS C., LUQUE L., MURILLO P. 
2081

Titulo: PROBLEMÁTICA ESTOMATOLOGICA EN EL COMPLEJO CARCELARIO "REVERENDO FRANCISCO LUCHESSE"

Autores: PELIZZON MORENO L., PANICO R., BRUZAM., FRANCIAC., ZAMITTO G., SEARA M.

2082

Titulo: FORMACIÓN DE RRHH COMO ESTRATEGIA ANTIESTIGMATIZADORA EN LA IMPLEMENTACIÓN DE LEYES DE SALUD MENTAL

Autores: DÁVILA A., ZARATE J., ILLANES M., SANANEZ G., SANCHEZ C., HUNZIKER C., GIOACCHINI C., PLANTÉ J.

\section{3}

Titulo: DETERMINANTES SOCIOECONÓMICOS DEL GASTO EN ALCOHOL DE LOS HOGARES INDÍGENAS DE LA ZONA URBANA DEL ALTIPLANO BOLIVIANO

Autores: CARRAZANA RIVERAA., DE SANTIS M., MOSCOSO N.

2084

Titulo: PERSPECTIVAS DE ACTIVIDADES DE PROMOCIÓN Y PREVENCIÓN DE DENGUE EN CIUDAD DE CÓRDOBA EN OCTUBRE 2016

Autores: GORDO M., CARBONETTI M., CARBONETTI M., COMBINA V.

2085

Titulo: VALORACIÓN DE ACTIVIDADES DE PROMOCIÓN Y PREVENCIÓN DE DENGUE CIUDAD DE CÓRDOBA DURANTE OCTUBRE 2016

Autores: GORDO M., CARBONETTI M., CARBONETTI M., COMBINA V.

2086

Titulo: ACEPTACIÓN DE ACTIVIDADES DE PROMOCIÓN Y PREVENCIÓN DE DENGUE EN CÓRDOBA DURANTE OCTUBRE DE 2016

Autores: GORDO M., CARBONETTI M., CARBONETTI M., COMBINA V.

2087

Titulo: DISTRIBUCIÓN DE LA ENFERMEDAD DE CHAGAS EN ADULTOS DE ÁREAS RURALES DEL CHACO SANTAFESINO

Autores: MENDICINO D., COLUSSI C., STAFUZA M., ACHKAR G., MANATTINI S., GARNERO N., MONTEMAGIORE S., NEPOTE M. 
2088

Titulo: CUIDADOS DE ENFERMERÍA OFRECIDOS POR PROFESIONALES EN SERVICIOS DE SALUD ESTATAL. CAPITAL. SANTIAGO DEL ESTERO - DESAFÍOS PARA LA SALUD PÚBLICA

Autores: ARIAS D., MEZQUIDA A., LOBOS M., REVAINERA M., PAZ TULIS M., QUIROGA M., CILENTO E., CAMPOS A.

2090

Titulo: SALUD AUDITIVA Y CALIDAD ACÚSTICA DE AULAS, DTU EN ALUMNOS DE NIVEL PRIMARIO EN CORDOBA

Autores: LUCCA M., ASSUM A., SCHAFER H., ABADIA L., PAVLIK M., MARISTANYA.

\section{1}

Titulo: FORTALECIMIENTO DE LAATENCIÓN PRIMARIA DE LA SALUD EN EL NOROESTE DE LA PROVINCIA DE CÓRDOBA, ARGENTINA. LA EXPERIENCIA DEL PROGRAMA REDES CÓRDOBA

Autores: BULLANO F., DUCANT M., HERRANDO P., JANISZEWSKI V., MALAJOVICH U., MILDENBERGER L., PEREZ L., RIBOLZI L., GUAZZINI C.

\section{3}

Titulo: EL IMPACTO DE LA ENCEFALITIS HERPÉTICA EN EL DESEMPEÑO NEUROCOGNITIVO

Autores: CISNEROS L., BASTIDA M., MIAS C.

2094

Titulo: AYUDANDO A CRECER CON SALUD

Autores: ORPHEE C., CRUZ R.

2096

Titulo: IMPACTO DE UN MODELO DE INTERVENCION EN ADULTOS MAYORES, VILLA DE LA QUEBARADA, SAN LUIS

Autores: TEJADA G., CLAVELES CASAS F., PIRAN ARCE F.

2097

Titulo: CONTROL DE AGUA DE CONSUMO EN ESCUELAS DE LA PROVINCIA DE TUCUMAN

Autores: CRUZ R., ORPHEE C.

Titulo: ESTADO NUTRICIONAL Y GANANCIA DE PESO DE EMBARAZADAS ASISTIDAS EN CENTROS DE SALUD.SALTA CAPITAL.

Autores: CONTRERAS N., BORELLI M., MAYORGA M., DE LA VEGA S., PASSAMAI M. 
2099

Titulo: NUNCA HABRÁ UN MAÑANA SIN AGUA, PROTEJAMOSLA

Autores: CHACON I., CORTEZ C., DIAZ A., GODOY M., MASSILILLA A., OREZZO M., RUIZ M., PIRAN ARCE M.

2102

Titulo: RELACIÓN ENTRE DETERMINANTES SOCIOCULTURALES Y MATERNIDAD COMO PROYECTO DE VIDA EN JÓVENES EMBARAZADAS O MADRES

Autores: PUMA T.

2103

Titulo: PERTENENCIA A UN PROGRAMA Y LA REALIZACIÓN DEL PAP EN UN CENTRO DE SALUD, SALTA

Autores: ECHALAR S., FARFAN A., FONTEÑES S.

2104

Titulo: PERFIL DEL BAJO PESO AL NACER EN LA MORTALIDAD INFANTIL. PERIODO 2015-16 PROVINCIA DE SALTA

Autores: ECHALAR S., APARICIO S.

2107

Titulo: LA EXTENSIÓN UNIVERSITARIA COMO HERRAMIENTA PARA LA PREVENCIÓN EN SALUD EN LA ADOLESCENCIA

Autores: SÁNCHEZ L., MARTINEZ L., CAMPANAV., CREMONEZZI C., ASIS O.

2108

Titulo: RELATO DE EXPERIENCIA

Autores: FRANCOLIN L., MARTINEZ R., SAVOY F., COSTA P., BELMONTE V.

2109

Titulo: PROYECTO: RECOBA VILLAGUAY

Autores: GERVASONI A., MICHEL J., CABANNA G., PIACENZA G., GILBERTO., WENDLER ., TORREZ M., RODRIGUEZ E., HORISBERGER G., ETCHEVERRY G., CANOVAS J., AZARIO R., BELMONTE V.

2110

Titulo: PROBLEMÁTICA DEL TABAQUISMO EN ADOLESCENTES. ACTIVIDAD DE EXTENSIÓN UNIVERSITARIA EN DOS ESCUELAS DE PROVINCIAS DIFERENTES

Autores: SANCHEZ L., MARTÍNEZ L., CAMPANAV., CREMONEZZI C., ASIS O 
2112

Titulo: POTENCIAL INFLAMATORIO DE LA DIETA Y SU ASOCIACIÓN CON LA FORMACIÓN DE PLACA DE ATEROMA

Autores: JUAN M., KRONWITTER B., NICLIS C., MUÑOZ S.

2113

Titulo: MEDIOS DE TRANSPORTE Y SINIESTRALIDAD VIAL EN LA PROVINCIA DE LA RIOJA EN EL PERIODO 2011- 2016

Autores: ANDRADA M., BERTONE C., TORRES V.

\section{4}

Titulo: COMUNICANDO FEDERALMENTE EN SALUD: LA EXPERIENCIA DEL CANAL SALUD

Autores: RIBETTI D., FERRER J., EYNARD M.

2124

Titulo: LA INTERCONSULTA CON PSICOLOGÍA MÉDICA EN UN HOSPITAL PEDIÁTRICO: UNA BÚSQUEDA DE ABORDAJE INTEGRAL

Autores: MORALES N.

HOSPITAL PEDIÁTRICO, CENTRO HOSPITALARIO PEREIRA ROSSELL, MONTEVIDEO, URUGUAY

2125

Titulo: FUNCIONES EJECUTIVAS Y RENDIMIENTO ESCOLAR: SU RELACION CON DIFERENTES CONTEXTOS SOCIOECONÓMICOS FAMILIARES

Autores: GRANDIS A., UVAA., DELGADO V., TARDITI L.

2127

Titulo: PROGRAMA DE PROMOCIÓN DE CUIDADOS DE SALUD EN LA LOCALIDAD DE BIALET MASSÉ. PROYECTO: PROMOTORES DE SALUD ADOLESCENTE

Autores: CEVILAN S., MEDINA M., ABREGÚ C., DEMIN N.

2128

Titulo: PROGRAMADE SALUD MENTAL COMUNITARIA(PSMC): DESAFIOS Y COMPLEJIDADES

Autores: BULACIO A., ROLFO F., ALMADA BONFIGLI D.

2132

Titulo: EL FENÓMENO DEL MALTRATO INFANTIL Y SU INTERVENCIÓN DESDE UNA RED INTERINSTITUCIONAL

Autores: PRAT R. 
Titulo: SENSIBILIDAD QUÍMICA MÚLTIPLE ASOCIADAA CONTAMINACIÓN AMBIENTAL EN UN BARRIO DE CIUDAD DE CÓRDOBA

Autores: AVILA-VAZQUEZ M., MATURANO E., DIIFLIPPO F., MACLEAN B.

2134

Titulo: SITUACION ALIMENTARIA Y ESTADO NUTRICIONAL DE LOS NIÑOS DE LA CIUDAD DE SAN LUIS

Autores: SOSAN., CORREAM., PIRANARCE M., GOMEZM., BESSEGACUADROS V., VEGAS., PAEZ G., SCATENA S., JUNCO MANSUR B.

2137

Titulo: HOSPITALES GENERALES: HACIA LA ADECUACIÓN DE LA ATENCIÓN EN SALUD MENTAL

Autores: CAMINADA ROSSETTI M., DEL CARPIO S.

2138

Titulo: FORTALECIENDO LOS VÍNCULOS ENTRE ADULTOS Y JÓVENES DEL IPEMYT 23 ANEXO-ARGÜELLO LOURDES

Autores: CARRIZO A.

\section{1}

Titulo: PROYECTO DE DISTRIBUCIÓN DE OPIOIDES PARA EL MANEJO DEL DOLOR POR CÁNCER EN HOSPITALES PÚBLICOS DE LA PROVINCIA DE BUENOS AIRES

Autores: TORRESI M., BUSTAMANTE C., HIDALGO M., SANTA MARIA J., VINIEGRA M.

2145

Titulo: PROMOCION DE MEDICAMENTOS GENERICOS: UNAALTERNATIVA CONVENIENTE Y EFECTIVA

Autores: DE LEONARDI A., VIRGA C., AGUZZI A., DE LEONARDI G.

2146

Titulo: EDUCACIÓN SOBRE MEDICAMENTOS EN BENEFICIO DE LA SALUD POBLACIONAL

Autores: DE LEONARDI A., VIRGA C., AGUZZI A., DE LEONARDI G. 
2149

Titulo: DIÁLOGO/S DE PRÁCTICAS COTIDIANAS DE CIUDADANÍA EN LA GENERACIÓN DE OPORTUNIDADES EDUCATIVAS Y DE CUIDADO

Autores: AIMAR Á., AHUMADA D., CLEMENTE C., DE DOMINICI C., TORRE M., VIDELA N., BURIN M., VEGA S., FONSECAA., ANDRADA C.

2151

Titulo: COMPARTIENDO HABILIDADES DE CO-CONSTRUCCIÓN DE ESPACIOS PARA LA INVESTIGACIÓN-ACCIÓN

Autores: AIMAR Á., AHUMADA D., CLEMENTE C., DE DOMINICI C., TORRE M., VIDELA N., BURIN M., VEGA S., FONSECAA.

2152

Titulo: SITUACIÓN SANITARIA DE LOS MENORES DE 6 AÑOS QUE ASISTEN AL CENTRO DE SALUD

Autores: HENZE G., RANAIVORISOA M., SCHELLER V., VERON D.

2160

Titulo: CAPACITACIÓN DE PROFESIONALES DE LA SALUD NUCLEADOS EN AMPROS EN SALUD MENTAL Y DISCAPACIDAD

Autores: NADAL D.

\section{1}

Titulo: IMPLEMENTANDO SIMULACIÓN COMO HERRAMIENTA EDUCATIVA EN UN AMBIENTE SEGURO, EN LA ASIGNATURA: ENFERMERÍA EN EL CUIDADO DEL ADULTO Y EL ANCIANO"

Autores: CARDOZO D., RIOS L.

2162

Titulo: PROMOVIENDO LA PREVENCIÓN DE FACTORES DE RIESGOS, EN LA COMUNIDAD EDUCATIVA DEL NIVEL PRIMARIO Y SECUNDARIO Autores: CARDOZO D., RÍOS L.

2164

Titulo: EL FENÓMENO DEL MALTRATO INFANTIL Y SU INTERVENCIÓN DESDE UNA RED INTERINSTITUCIONAL

Autores: PRAT R.

2166

Titulo: EMERGENCIA DE HIPOTIROIDISMO EN UNA LOCALIDAD AGRÍCOLA DE LA PROVINCIA DE CÓRDOBA

Autores: ÁVILA-VAZQUEZ M., MATURANO E., ETCHEGOYEN A., DIFILIPPO F., MACLEAN B. 
Titulo: PANORAMA LATINOAMERICANO DE LA FORMACION DOCTORAL EN ENFERMERÍA

Autores: PALACIO J., COMETTO M., GOMEZ P., MALVÁREZ S.

2170

Titulo: INCIDENCIA DE BAJO PESO AL NACIMIENTO EN RELACIÓN A LA ALTURA GEOGRÁFICA EN JUJUY 1991-2013

Autores: REVOLLO G., ALFARO E., DIPIERRI J., DÍAZ M.

\section{3}

Titulo: PROYECTO DE PROMOCIÓN Y PREVENCIÓN DEL BULLYING ESCOLAR EN UNA INSTITUCIÓN PÚBLICA DEL GRAN BUENOS AIRES

Autores: GARAY G., AVENDAÑO V., TORRES M.

2174

Titulo: MITOS Y CREENCIAS QUE INFLUYEN EN EL COMPORTAMIENTO SEXUAL DE EMBARAZADAS

Autores: LEIVA C., SCHULER E., MURAVCHIK B., BAUCERO C., TÁLAMO F., LIJSTENTEIN M., GORISCHNIK R., GUARISCHI N., VIERA N., LEGUIZA J., GODOY M., ROZALES M.

2175

Titulo: CALIDAD DE VIDAYAPOYOSOCIALEN PACIENTES INTERVENIDOS CON CÁNCER COLORRECTAL

Autores: FERRAZIE E., MOUSSOU M.

2177

Titulo: EXPOSICIÓN A PESTICIDAS Y SUSTANCIAS INORGANICAS COMO CAUSANTES DE LA PROTEINOSIS ALVEOLAR PULMONAR

Autores: ERNST G., ROBAINA G., GOMEZ T., YOUNG P.

2180

Titulo: ESTRÉSACADÉMICOYCONSUMODESUSTANCIAS PSICOACTIVAS EN ALUMNOS INGRESANTES A LA CARRERA DE ODONTOLOGÍA, UNIVERSIDAD NACIONAL DE CÓRDOBA

Autores: RODRÍGUEZ I.

2182

Titulo: UNIDAD DE GESTIÓN DE POLÍTICAS PREVENTIVAS DE VIOLENCIA FAMILIAR Y DE GÉNERO

Autores: ALCORTA A., MORALES P., PEREYRA M. 
2187

Titulo: ASOCIACIÓN ENTRE LA INFECCIÓN POR VPH Y EL CONSUMO DE MATE COMO FACTORES DE RIESGO PARA EL CÁNCER BUCAL. ESTUDIO PRELIMINAR

Autores: ZAPATA M., CRISCUOLO I., BOLESINA N., ALLENDE A., ROBLEDO G., MORELATTO R.

2189

Titulo: ESTUDIOS DE BIODISPONIBILIDAD Y BIOEQUIVALENCIA

Autores: FARFÁN S., DABBENE V., CASTELLI G., QUINZIO E., GARCÍA V., SCARELLO A.

2190

Titulo: ROL DE LA DIVULGACIÓN Y TRANSFERENCIA EN UN PROGRAMA DE EXTENSIÒN DIRIGIDO A ESTUDIANTES SECUNDARIOS

Autores: ROSALES F., ASIS O., LUQUE C., FONSECA I., SPITALE L.

2193

Titulo: LASALUD DE LA POBLACIÓN, DE UNA COMUNIDAD DEL NORESTE CORDOBES. CÓRDOBA. ARGENTINA

Autores: MIRANDA F., BERTONE C.

\section{4}

Titulo: CALIDAD DE VIDA ORAL EN PACIENTES VIH POSITIVO

Autores: STROPA G., ZOELA V.

2195

Titulo: CENTRO DE FARMACOVIGILANCIA MATERNO-NEONATAL

Autores: GUERRERO C.

2196

Titülo: APROXIMACIÓN A LA INVESTIGACIÓN EN LA FORMACIÓN DE ESTUDIANTES DE LAS PRÁCTICAS EN SALUD PÚBLICA

Autores: BARBERO L., MARTINA D., SCRUZZI G., EPPENS E., AGOSTINA G., BALMACEDA N., ULLOQUE J., QUIÑONES V.

2197

Titulo: PERFIL DE SALUD DE COMUNIDADES EXPUESTAS CRÓNICAMENTE A AGROQUÍMICOS

Autores: BERTONE C., ANDRADA M., DELGADO M., AGOST L., MIGUEL C., MIRANDA F. 
Titulo: REPORTE DE CASO: INTERACCIÓN MEDICAMENTOSA ENTRE CICLOSPORINA Y FENITOÍNA. RELEVANCIA CLÍNICA

Autores: GONZALEZ I., PEREZ M., MARKS L., RIVOLTAS., SUAREZ H.

2199

Titulo: CARACTERIZACIÓN DE LAS INTOXICACIONES PEDIÁTRICAS CON MONÓXIDO DE CARBONO EN EL HOSPITAL DE NIÑOS DE LA SANTÍSIMA TRINIDAD

Autores: MARKS L., GONZALEZ I., ODIERNA E., RIVOLTA S., SUAREZ H.

2200

Titulo: RIESGOS PSICOSOCIALES DE LA POBLACIÓN DE ENFERMEROS PROFESIONALES DE LOS SERVICIOS PENITENCIARIOS DE LA PROVINCIA DE CÓRDOBA, ARGENTINA. (2016 - 2017)

Autores: NIEVAS S., GOMEZ P., COMETTO M.

2201

Titulo: APROXIMACIÓN A LA INVESTIGACIÓN EN LA FORMACIÓN DE ESTUDIANTES DE LAS PRÁCTICAS EN SALUD PÚBLICA

Autores: BARBERO L., MARTINA D., SCRUZZI G., EPPENS M., ULLOQUE J., GARCIAA., BALMACEDA N., ACOSTAA.

2202

Titulo: ESPACIOS DE CONSEJERÍA: APROXIMACIONES A LA LACTANCIA MATERNA EN CENTROS DE SALUD BASADOS EN APS

Autores: BARBERO L., MARTINAD., EPPENSM., SCRUZZIG., ULLOQUEJ., GARCIA A., BALMACEDA N., ACOSTAA., GENTI M., QUIÑONES V.

2203

Titulo: CONFIGURACIÓN GEOGRÁFICA DE LA MORTALIDAD POR ENFERMEDADES NO TRANSMISIBLES EN ARGENTINA: APORTES METODOLÓGICOS EN SALUD

Autores: POU S., TUMASN., SÁNCHEZSORIAD., BECARIACOQUET J., DÍAZM.

2204

Titulo: PREVENCIÓN DEL SÍNDROME URÉMICO HEMOLÍTICO: PROPUESTA EDUCATIVA DESDE LA UNIVERSIDAD NACIONAL DE CÓRDOBA

Autores: PASSERA P., SARTOR S., MONTANI L., VIOLA L. 
2208

Titulo: IDENTIFICACIÓN Y FORTALECIMIENTO DE HÁBITOS DE AUTOCUIDADO DE SALUD EN NIÑOS DE ESCUELA PRIMARIA

Autores: CHALUP E., BONNIN C., OROZCO M.

2209

Titulo: LA SALUD EN BARRIO LOS CORTADEROS. INTERVENCIÓN INTERDISCIPLINARIA DESDE UNA TECNICATURA EN ENFERMERÍA

Autores: ALCORTAA., GUZZI L., MAMONDE V., CAMPANELLO S.

\section{1}

Titulo: LACASAS FUNGICAS $Y$ SU EXPLORACION PARA LA CLARIFICACION DE JUGOS DE MANZANA

Autores: GUERBEROFF G., DE ELIAS M., RUIZ POSSE A., CAMUSSO C.

2212

Título: GRADO DE SATISFACCIÓN DE LA ATENCIÓN ODONTOLÓGICA RECIBIDA POR ESTUDIANTES DE LA UNC

Autores: SICA SANCHEZ M., SANCHEZ DAGUM M.

2215

Titulo: PÉRDIDA DE LOS PRIMEROS MOLARES PERMANENTES EN ADOLESCENTES

Autores: SICA SANCHEZ M., ALCAZAR V.

2218

Titulo: PROCESO DE ALIMENTACIÓN-NUTRICIÓN Y DESARROLLO SOSTENIBLE

Autores: MARTINA D.

2219

Titulo: MEDICAMENTOS DEALTO RIESGO SANITARIO: ABORDAJE DESDE LA FARMACIA INSTITUCIONAL EN UN HOSPITAL PÚBLICO

Autores: GARCIA V., CHICHALOF C.

2220

Titulo: FORTALECIMIENTO DE REDES INTEGRADAS DE VIGILANCIA DE LA SALUD EN LA PROVINCIA DE CÓRDOBA. 2016

Autores: BARBAS M., CANNA F., WILLINGTON A., VILLACÉ M, CEVILÁN S., RODRÍGUEZ JUNYENT C. 
Titulo: RELATO DE EXPERIENCIAS EN ZONAS NO IRRIGADAS DE LAVALLE

Autores: GARCÍA CARDONI M., ZABALA W., HIRAMATSU T., GARCÍA CRIMI G., HELLWIG G., GALDEANO F., SICILIAA., OLIVARES C., ARGÜELLO D.

2223

Titulo: DISEÑO DE LA CONFIGURACIÓN EDILICIA DE UNA FARMACIA HOSPITALARIA BASADA EN EL CUMPLIMIENTO DE NORMATIVAS

Autores: BUSTOS FIERRO C., SOBRERO C.

2224

Titulo: INTERNACIONES DE ADOLESCENTES CON CONSUMO DE SUSTANCIAS. UN DEBATE NECESARIO

Autores: MORAN R.

2225

Titulo: EL APORTE DE LA INTERCULTURALIDAD EN EL ABORDAJE DE LAS PRÁCTICAS DE CUIDADO NUTRICIONAL INFANTIL

Autores: GARCÍAA., BARBERO L., MARTINA D

2226

Titulo: UTILIZACIÓN DE GOMA BREA EN PAN PARA CELÍACOS Y SU RELACIÓN CON LA SEGURIDAD ALIMENTARIA

Autores: PIRAN ARCE M., CLAPASSON P., MERINO N., CAMPDEROS M., GONZALEZ U., RINALDONI N.

2228

Titulo: ENFERMEDADES NO TRANSMISIBLES Y FACTORES DE RIESGO. ARGENTINA, 2013

Autores: BERTONE C., ALVAREZ M.

2229

Titulo: DENGUE, ZIKA, CHIKUNGUYA. IMPORTANCIA EN EL BANCOS DE SANGRE DE LA UNIVERSIDAD NACIONAL DE CORDOBA

Autores: CARABAJAL MIOTTI C., GONZALEZ SILVA N., FRATTARI S.

2230

Titulo: FACTORES DE RIESGO AMBIENTALES EN RELACIÓN AL ESTADO DE SALUD DE LA POBLACIÓN DE SAN LUIS

Autores: PIRAN ARCE M., STAURINI S., LIMA M. 
Titulo: DESAFíO PENDIENTE: OTRA EDUCACIÓN, OTRO SISTEMA DE SALUD EDUCACIÓN PERMANENTE EN CENTROS DE ATENCIÓN PRIMARIA

Autores: ROLLÁN R., VILLARREAL L., RABOY E., BOCCANERA V., COLELLA P., CLOSA C., GALLARDO C., JOZAMI A., WEIHMULLER A.

\section{4}

Titulo: DESAFÍO PENDIENTE: OTRA EDUCACIÓN, OTRO SISTEMA DE SALUD EDUCACIÓN PERMANENTE EN CENTROS DE ATENCIÓN PRIMARIA

Autores: ROLLAN R., VILLARREAL L., RABOY E., BOCCANERA V., COLELLA P., CLOSA C., GALLARDO C., JOZAMI A., WEIHMULLER A.

2236

Titulo: DESARROLLO SISTEMA DE NAVEGACIÓN DE PACIENTES ONCOLÓGICOS PROV. BS AS.

Autores: GARCIA DE LAS LONGAS M., GUARNIERI RONDINA S., SERRANO M., LEUENBERGER M., VINIEGRA M.

2237

Titulo: DIAGNÓSTICO Y PROMOCIÓN DE LA SALUD BUCAL DE NIÑOS CON DISCAPACIDAD QUE ASISTEN A CINCO ESTABLECIMIENTOS DE EDUCACIÓN ESPECIAL DE CÓRDOBA

Autores: HERRERAA., JEREZ M., LAGONERO A., MARTINEZ M., CARAMELLO A., DE JUANA D., OLIVA M., MARTINEZ L., PIRE M., ROCAMUNDI M., ALBRECHT V., ULLOQUE J

2238

Titulo: NIVELES DE EMPATÍA EN ESTUDIANTES DE ODONTOLOGÍA DE LA UNIVERSIDAD CATÓLICA DE CÓRDOBA, ARGENTINA

Autores: VARELAT., ULLOQUE J., VILLALBAS., VILLALBAR., DIAZ NARVAEZP.

2239

Titulo: ATENCIÓN PRIMARIAAMBULANTE DE SALUD EN TRASLASIERRA, CORDOBA

Autores: CHALUP M., BONNIN C., BERTORELLO N., GARCIA FERREYRA S.

2240

Titulo: APLICACIONES MÓVILES PARA REDUCIR COSTO DE LAATENCIÓN PRIMARIA

Autores: SIMOES L., HEREDIA R. 
2241

Titulo: FARMACOVIGILANCIA EN EL TRASTORNO BIPOLAR. LAMOTRIGINA

Autores: PAZ SOSA M., GUERRERO C., CORRALES R.

2242

Titulo: FLEXSENSING - UNA SOLUCIÓN PARA REDUCIR COSTO DE LA ATENCIÓN PRIMARIA

Autores: SIMOES L., HEREDIA R.

2243

Titulo: EVALUACIÍN DEL CONOCIMIENTO SOBRE VIH-SIDA EN ESTUDIANTES DE LA REGIÓN DEL VALLE DE LERMA. SALTA, ARGENTINA. 2016

Autores: FARFÁNA., FARFÁN C., BERTAD., GLOBERT M., ROMERO S., AVILAF.

2244

Titulo: RIESGO CARDIOVASCULAR, CARACTERIZACIÓN DE ADULTOS QUE ASISTEN A UN CENTRO DE SALUD DE ZONA OESTE, SALTA, ARGENTINA

Autores: ROMERO S., VILCA G., FARFÁN A., MILLÁN M., VARGAS C.

2245

Titulo: DETERMINANTES DE LAS MUERTES MATERNAS PERÍODO 201506. PROVINCIA DE SALTA

Autores: ECHALAR S., APARICIO S

2247

Titulo: INTERVENCIÓN PARA REDUCIR AL AUTOESTIGMA CON USUARIOS DE UN SERVICIO AMBULATORIO DE SALUD MENTAL EN CHILE

Autores: BUSTAMANTE J.

2248

Titulo: SITUACIÓN LABORAL DE LOS LIC. EN NUTRICIÓN DEL SECTOR PÚBLICO DE LOS MUNICIPIOS CORDOBESES

Autores: BARBERO L., EPPENS M.

2250

Titulo: TALLER DE ADHERENCIA AL TRATAMIENTO ANTIRRETROVIRAL EN EL HOSPITAL DE NIÑOS DE CÓRDOBA

Autores: GARNERO A., MIGUELTORENA E., SANTIAGO A., ALLENDE G., GOMILA A., VANZO C., PIEDRA D., PICCO A., PERSONENNI P. 


\section{1}

Titulo: DETERMINACIÓN DEL CONOCIMIENTO DEL MARCO JURÍDICOREGULATORIO DE HISTORIA CLÍNICA ODONTOLÓGICA

Autores: STROPA G., BAGLINI C., ZAMAR S., TOMAS D.

2252

Titulo: EVALUACION DE CONOCIMIENTOS SOBRE VIAS DE TRANSMISIÓN DE VIH-SIDA EN ESTUDIANTES DE SALTA. ARGENTINA. 2016

Autores: GILOBERT M., AVILA F., BERTA D.

\section{5}

Titulo: CONDUCTAS Y FUENTES DE INFORMACIÓN DE VIH-SIDA EN ESTUDIANTES DE SALTA. ARGENTINA. 2016

Autores: GILOBERT M., AVILA F., BERTA D.

\section{6}

Titulo: DESAFÍO PENDIENTE: OTRA EDUCACIÓN, OTRO SISTEMA DE SALUD EDUCACIÓN PERMANENTE EN CENTROS DE ATENCIÓN PRIMARIA

Autores: ROLLAN R., VILLARREAL L., RABOY E., BOCCANERA V., COLELLA P., CLOSA C., GALLARDO C., JOZAMI A., WEIHMULLER A.

\section{8}

Titulo: FACTORES DE RIESGO CARDIOVASCULAR Y HÁBITOS DE VIDA EN EMPLEADOS DEL PODER JUDICIAL DE LA PROVINCIA DE CÓRDOBA

Autores: LÓPEZ A., PEREA H., MACHADO A., SCHLIAMSER L., RAMOS VILA A., FACCHIN H., RAPISARDI G., CECCHETTO E.

2260

Titulo: DESARROLLO DE UN PROGRAMA HÁBITOS SALUDABLES DESTINADO A PERSONAS CON SOBREPESO Y OBESIDAD

Autores: LÓPEZA., PEREA H., RAMOS VILAA., AGUIRRE A., VAGO E., ZELADA S., ANICETO M., DORDONI S.

2262

Titulo: DESARROLLO DE UN PROGRAMA DE CESACIÓN TABÁQUICA. VIAS CLIINICAS Y PROTOCOLOS

Autores: GUANA D., LÓPEZA., RAMOS VILAA., PEREA H., AGUIRRE A., VAGO E., SCHLIAMSER L. 


\section{0}

Titulo: UNIENDO REDES EN MATERNIDAD E INFANCIA

Autores: BRILO R, CONTATO A, GIUSTI G, MONTENEGRO F, MORALES G, ROMERO M L, SCHENK V, YANOVER $M$.

3001

Titulo: CUERPOS Y SUBJETIVIDADES ENTRE TEATRO ANATÓMICO Y ROBÓTICA. (DES) MONTAJE TRANSDICIPINAL, TRANSCIONAL Y DECOLONIAL

Autores: COTAIMICHV, GENEROSO K, ALVAREZ C

3003

Titulo: FORMACIÓN EN SALUD MENTAL Y ADULTOS MAYORES

Autores: AMBROSI J, SAMMAN M

3008

Titulo: EL ENFERMERO EN VALORES HUMANÍSTICOS

Autores: RODRIGUEZ R, ALARCON A, CONDORI N, GIL FERNANDEZ M, QUISPE G, LOPEZ P

3009

Titulo: PERIL SOCIO EPIDEMIOLÓGICO DE LAS MADRES QUE SE HOSPEDARON EN LA RESIDENCIA DE MADRES (RM) CUYOS BEBES PREMATUROS FUERON INTERNADOS EN NEONATOLOGÍA Autores: GONZALEZ G, PEREYRA G, TOLEDO M, MELGAREJO S

3011

Titulo: INFORMACIÓN Y ACEPTABILIDAD DE LA VACUNA CONTRA HPV EN MÉDICOS GENERALES DE FAMILIA Y GINECÓLOGOS QUE SE DESEPEÑAN EN APS DE LA MUNICIPALIDAD DE CBA

Autores: NIÑO C. C.

3012

Titulo: CARACTERIZACIÓN DEL PROGRAMA RASTREO DEL CÁNCER CERVICO- UTERINO DEL PRIMER NIVEL DE ATENCIÓN MUNICIPAL DE CÓRDOBA

Autores: AVILA S.

3014

Titulo: NAVEGACIÓN EN CANCER DE MAMA: REGISTRO, TRANSFORMACIONES Y TRAYECTORIAS COMPLEJAS

Autores: ROBLES N, PIEDRABUENA M.C, LIBOIS I, GIUNTA C, MOREIRA M, GRANDA P, PESCE V. 
3015

Titulo: PREVENCIÓN DE CAÍDAS Y CAPACIDAD FUNCIONAL EN EL ADULTO MAYOR

Autores: MARTINEZ H.D, FERRER VERA M, JURI G. A, ZANETTA V. H, CASTRO TOSCHI R.

3016

Titulo: GESTIÓN COLECTIVA EN SALUD, LO QUE EL AGUA SE LLEVÓ

Autores: CUEVAS AV, MANGUPLI E, DIAZA J.A., GUARNERI R, FARIA V, CABALLÉ MR, APÓSTOLO A, CIUFFOLINI MB, AVILA S.

3017

Titulo: ACTUANDO EN EL PRESENTE, PENSANDO EN EL FUTURO. NECESIDADES EDUCATIVAS ESPECIALES Y COMUNIDAD

Autores: BOSSIO A.

3018

Titulo: MODELO DE INTERVENCIÓN EN SITUACIONES CRÍTICAS A PERSONAS CON DIAGNÓSTICO DE PSICOSIS: UNA MIRADA DESDE EL EQUIPO CLÍNICO

Autores: COMETTO MC, GOMEZ P, ACUÑA R, MAZZONI E, ALVARADO R, VALENCIA E., FERNANDEZ R.

3019

Titulo: MODELO DE INTERVENCIÓN EN SITUACIONES CRÍTICAS A PERSONAS CON DIAGNÓSTICO DE PSICOSIS: LA RELACIÓN DIALÉCTICA ENTRE PARES Y USUARIOS

Autores: COMETTO MC, GOMEZ P, CÓRDOBA S, SCOTTO V, PAIVA A, ALVARADO R, VALENCIA E, FERNANDEZ R. Subsidio NIMH 1 UI9MH095718

3020

Titulo: EVALUACIÓN DE LA ATENCIÓN DE LOS SERVICIOS DE SALUD MENTAL DESDE LA PERSPECTIVA DE LOS USUARIOS

Autores: GONZALEZ F, ROMERO D, ALVARADO R, FERNANDEZ R. Subsidio $\mathrm{NIMH} 1 \mathrm{UI} 9 \mathrm{MH} 095718$

3021

Titulo: EVALUACIÓN DEL COMPONENTE PSICO-SOCIO-CULTURAL EN USUARIOS DEL SISTEMA DE ATENCIÓN EN SALUD MENTAL

Autores: LUCCHESE M, BONO M, CHAUBET M, LUQUE C, VALENCIA E, FERNÁNDEZ R. Subsidio NIMH 1UI9MH095718 
3022

Titulo: CAPACITACIÓN EN PROCESO DE INVESTIGACIONES ETNOGRÁFICAS EN EL ÁREA DE SALUD MENTAL.

Autores: LUCCHESE M, BONO M, CHAUBET M, LUQUE C, COCA S.M.

Subsidio NIMH 1 UI9MH095718

3023

Titulo: EL MILAGRO DEL SAUCE SALUD TERRITORIAL RELEVAMIENTO SOCIO- SANITARIO Y PROMOCIÓN CULTURAL DE CARÁCTER PARTICIPATIVO

Autores: COTAIMICH V.

3024

Titulo: PRÁCTICAS INNOVADORAS EN EL SERVICIO DE ENFERMERÍA I.P.A.D, INSTITUTO PROVINCIAL DE ALCOHOLISMO Y DROGADICCIÓN. CORDOBA

Autores: CATALINI, S

3025

Titulo: LA EDUCACIÓN PERMANENTE COMO HERRAMIENTA PARA IMPLEMENTAR SALAS DE SITUACIÓN AÑO 2016

Autores: PEISINO E, MARTI M, AMMAN C, GAUNA D, ACEVEDO G

3026

Titulo: VIOLENCIA DE GÉNERO Y FEMICIDIO

Autores: BUNDIO R, GOMEZ T, SEGUEL I.

3027

Titulo: CUESTIONARIO DE OPINION PARA EVALUAR EL CONOCIMIENTO DEL IMPACTO DE LA CONTAMINACION DEL RIACHUELO

Autores: SALDIVAR I, CHAMBI T, ROSALES S, CONDARSO J, ARIAS CUBAS B, VALLEJOS E.

3503

Titulo: ESTIMACIONES DE LAS TENDENCIAS HISTÓRICAS DE DESASTRES EN MÉXICO, A PARTIR DE LA BASE DE DATOS EMDAT.

Autores: VÁSQUEZ AVENDAÑO B., COCA S.M., ABELDAÑO ZÚÑIGA R.A. UNIVERSIDAD DE LA SIERRA SUR. OAXACA, MÉXICO. 
3504

Titulo: CALIDAD DE VIDA RELACIONADA A LA SALUD EN USUARIOS DE SERVICIOS DE SALUD MENTAL DE LA CIUDAD DE CÓRODBA.

Autores: BURRONE M. S., ABELDAÑO R. A., REGINATTO G. A., ROMERO D. E., ENDERS J. E., ALVARADO R., FERNÁNDEZ R.

UNIVERSIDAD NACIONAL DE CÓRDOBA

Subsidio NIMH 1 UI9MH095718

2216

Titullo: ESTUDIO DE MORTALIDAD EN SOBREVIVIENTES DE TORTURA Y PRISION POLITICA EN CHILE (1973-1990).

Autores: JORQUERA GONZALEZ M., MADARIAGA C.

3505

Titulo: CONOCIMIENTO DE LAS MUJERES EN LAS RECIDENCIAS PARA MADRES (RM) SOBRE METODOS ANTICONCEPTIVOS (MAC)

Autores: FERNÁNDEZ B., GONZÁLEZ G., TORRES M., YANOVER M. 